\title{
LHC Probes of TeV-Scale Scalars in SO(10) Grand Unification
}

\author{
Ufuk Aydemir and Tanumoy Mandal \\ Department of Physics and Astronomy, Uppsala University, P.O. Box 516, 75120 Uppsala, Sweden
}

Correspondence should be addressed to Tanumoy Mandal; tanumoy.mandal@physics.uu.se

Received 14 February 2017; Accepted 24 April 2017; Published 30 May 2017

Academic Editor: Anna Cimmino

Copyright (c) 2017 Ufuk Aydemir and Tanumoy Mandal. This is an open access article distributed under the Creative Commons Attribution License, which permits unrestricted use, distribution, and reproduction in any medium, provided the original work is properly cited. The publication of this article was funded by SCOAP ${ }^{3}$.

\begin{abstract}
We investigate the possibility of $\mathrm{TeV}$-scale scalars as low energy remnants arising in the nonsupersymmetric $\mathrm{SO}(10)$ grand unification framework where the field content is minimal. We consider a scenario where the $\mathrm{SO}(10)$ gauge symmetry is broken into the gauge symmetry of the Standard Model (SM) through multiple stages of symmetry breaking, and a colored and hypercharged scalar $\chi$ picks a TeV-scale mass in the process. The last stage of the symmetry breaking occurs at the TeV-scale where the leftright symmetry, that is, $\mathrm{SU}(2)_{L} \otimes \mathrm{SU}(2)_{R} \otimes \mathrm{U}(1)_{B-L} \otimes \mathrm{SU}(3)_{C}$, is broken into that of the $\mathrm{SM}$ by a singlet scalar field $\delta$ of mass $M_{\delta} \sim 1 \mathrm{TeV}$, which is a component of an $\mathrm{SU}(2)_{R}$-triplet scalar field, acquiring a TeV-scale vacuum expectation value. For the LHC phenomenology, we consider a scenario where $\mathcal{S}$ is produced via gluon-gluon fusion through loop interactions with $\chi$ and also decays to a pair of SM gauge bosons through $\chi$ in the loop. We find that the parameter space is heavily constrained from the latest LHC data. We use a multivariate analysis to estimate the LHC discovery reach of $\mathcal{S}$ into the diphoton channel.
\end{abstract}

\section{Introduction}

After the discovery of the Higgs boson at the Large Hadron Collider (LHC) [1,2], the last piece of the triumphant achievement of the high energy physics community, the Standard Model (SM), the great expectations for the observation of some sort of new physics at the LHC, emanated from the paradigms based on the familiar intuitions, some of which have so far lead the community to success, have turned out to be great disappointments as the LHC searches to date have returned empty-handed. Although there have been a couple of noticeable excesses, such as the diphoton $[3,4]$ (see [5] for a review and the full list of references) and diboson [6-8] anomalies, which caused excitement among the community, these signals have turned out to be statistical fluctuations as more data accumulates in.

While the LHC is still up and running and looking for any hint of trace pointing to physics beyond the SM (BSM), the community has been in an ambitious effort for projecting out the LHC implications of variety of new physics models for a possible future discovery. Among the various search channels, the diphoton resonance search is one of the most important programs at the LHC since this channel provides a comparatively cleaner background. One of the key predictions of many BSM theories is the existence of diphoton resonances around the $\mathrm{TeV}$-scale arising from the decay of $\mathrm{TeV}$-scale scalars present in those models.

One of the most appealing scenarios for a more fundamental picture is the Grand Unified Theory (GUT) framework, in which the $\mathrm{SO}(10)$ GUT is particularly interesting [924] (see [25-31] for analyses of the supersymmetric $\mathrm{SO}(10)$ GUT). Breaking the $\mathrm{SO}(10)$ gauge symmetry into that of the SM can be realized in a single step as well as in multiple steps by various symmetry breaking sequences. The relevant option we consider in this paper is the latter, while one possible intermediate phase, which we assume to be in the TeV-scale, is the left-right model whose gauge symmetry is based on $\mathrm{SU}(2)_{L} \otimes \mathrm{SU}(2)_{R} \otimes \mathrm{U}(1)_{B-L} \otimes \mathrm{SU}(3)_{C}\left(G_{2213}\right)$ [32-39], which is different than the left-right symmetric version since in this case $\mathrm{SU}(2)_{L}$ and $\mathrm{SU}(2)_{R}$ gauge couplings are different, that is, $g_{L} \neq g_{R}$. Adopting the minimalistic approach and, therefore, keeping the initial field content (the $\mathrm{SO}(10)$ multiplets) minimal, and tempted by the least possible fine-tuning intuition, it seems not possible to obtain a plausible scenario where the left-right model lies in the TeV-scale [23]. For instance, if the Higgs content is determined based on the extended survival 
hypothesis (ESH) [40], the model does not allow symmetry breaking scale of the left-right model to be in the TeV-scale. Recall that the ESH states that at every step of a symmetry breaking sequence, the only scalars which survive below the corresponding symmetry breaking scale are the ones which acquire vacuum expectation values (VEVs) at the subsequent levels of the symmetry breaking. However, by slightly relaxing the ESH conjecture by allowing one or more colored scalars to become light (at the TeV-scale), it is possible to have a TeVscale left-right model in the $\mathrm{SO}(10)$ framework [23].

In this paper, we investigate the phenomenology of $\mathrm{TeV}$ scale scalars as low energy remnants of the nonsupersymmetric $\mathrm{SO}(10)$ GUT. The part of the model that lies in the $\mathrm{TeV}$-scale, as mentioned above, is the left-right model, augmented by a color-triplet scalar $\Delta_{R}(1,3,2 / 3,3)$, whose one component $\chi$, we assume for our demonstration, has a mass of $\sim 1 \mathrm{TeV}$, while its other components are heavier in the $\mathrm{TeV}$ range. In particular, we explore the phenomenology of a SM-singlet scalar $\mathcal{S}$ of mass around $1 \mathrm{TeV}$ which is assumed to be the excitation of the neutral component of an $\mathrm{SU}(2)_{R}$ triplet $\Delta_{R_{1}}(1,3,2,1)$, denoted as $\Delta_{R_{1}}^{0}$. The field $\Delta_{R_{1}}^{0}$ breaks the symmetry of the left-right model into that of the SM by acquiring a VEV presumably at the TeV-scale in our set-up. The scalar $\chi$ is responsible for the production and decay of $\mathcal{S}$ through loop interactions.

In our model, we assume two intermediate energy scales between the electroweak scale $M_{Z}$ and the unification scale $M_{U}$. At the scale $M_{U}$, the $\mathrm{SO}(10)$ is broken into the PatiSalam group, $\mathrm{SU}(2)_{L} \otimes \mathrm{SU}(2)_{R} \otimes \mathrm{SU}(4)_{C}\left(G_{224}\right)$. The PatiSalam group is broken into the group of the left-right model at the first intermediate energy scale $M_{C}$, which is followed by the breaking of the left-right model into the SM at the energy scale $M_{R}$. In our scenario, $M_{R}$ is assumed to be in the TeVscale, while the values of $M_{U}$ and $M_{C}$ come out as predictions of the model. Note that the $D$-parity invariance $[10,11$, 41], which is a $Z_{2}$ symmetry that maintains the complete equivalence of the left and the right sectors, is broken together with the $\mathrm{SO}(10)$ in the first stage of the symmetry breaking. Therefore, the gauge couplings associated with the $\operatorname{SU}(2)_{L}$ and $\mathrm{SU}(2)_{R}$ gauge groups, $g_{L}$ and $g_{R}$, evolve under the influence of different particle contents; hence $g_{R} \neq g_{L}$, below the scale $M_{U}$. Remember that the $D$-parity is slightly different from the usual Lorentz parity in that the latter does not transform scalars, while the $D$-parity transforms them nontrivially. Note also that we remain in the minimal picture in terms of the total field content; the model does not have any extra matter field or any scalar $\mathrm{SO}(10)$ multiplet other than the ones required to begin with. Thus, the advantage of having a TeV-scale colored scalar is twofold: it is responsible for the production and decay of $\mathcal{S}$ and it can successfully be embedded in the minimal nonsupersymmetric SO(10) GUT scheme while maintaining the field content minimal.

In this paper, we identify the region of parameter space of our model constrained from the latest LHC data. By using a multivariate analysis (MVA), we compute the higher-luminosity LHC discovery reach of $\mathcal{S}$ into the diphoton channel where, as we will discuss later, the most stringent bounds come from.
The paper is organized as follows. In Section 2, we review the left-right model in the $\mathrm{SO}(10)$ grand unification framework. We discuss how the two scalars, $\mathcal{S}$ and $\chi$ of our interest, arise in our set-up. In Section 3, we discuss the unification of the couplings, derive the values of the intermediate symmetry breaking scales, and present the resulting predictions of the model. In Section 4, we present the phenomenology of $\delta$ and $\chi$ including the exclusion limits from the LHC data and future discovery prospects. We summarize our conclusions in Section 5.

\section{The Model}

We consider a left-right model, whose gauge group is $\mathrm{SU}(2)_{L} \otimes$ $\mathrm{SU}(2)_{R} \otimes \mathrm{U}(1)_{B-L} \otimes \mathrm{SU}(3)_{C}$, which is assumed to be broken into the $\mathrm{SM}$ at the $\mathrm{TeV}$-scale. The breaking is realized by the neutral component $\left(\Delta_{R_{1}}^{0}\right.$ which we denote as $\left.\mathcal{S}\right)$ of the $\mathrm{SU}(2)_{R}$ triplet $\Delta_{R_{1}}(1,3,2,1)$, which is commonly preferred in the literature. Here, instead of the $\mathrm{SU}(2)$ triplets, the $\mathrm{SU}(2)$ doublet $(1,2,1,1)$, which originates from the $\mathrm{SO}(10)$ multiplet 16, can also be used. The advantage of the triplet representation is that it can provide a Majorana mass term for the right-handed neutrino and, hence, the seesaw mechanism [42-46] for small neutrino masses.

In this work, we explore the phenomenology of the SMsinglet $\mathcal{S}$ which we assume to be produced and decayed through the loop interaction with a color-triplet hypercharged scalar $\Delta_{R_{3}}^{4 / 3}(1,8 / 3,3)$ denoted as $\chi \cdot \chi$ originates from the decomposition of $\Delta_{R}(1,3,2 / 3,3)$ component of the $G_{224}$ multiplet $\Delta_{R}(1,3,10)$ into the SM group as follows:

$$
\begin{gathered}
\Delta_{R_{3}}\left(1,3, \frac{2}{3}, 3\right)=\Delta_{R_{3}}^{4 / 3}\left(1, \frac{8}{3}, 3\right) \oplus \Delta_{R_{3}}^{1 / 3}\left(1, \frac{2}{3}, 3\right) \\
\oplus \Delta_{R_{3}}^{-2 / 3}\left(1, \frac{-4}{3}, 3\right) .
\end{gathered}
$$

For our purpose, we take the mass of $\chi$ around $1 \mathrm{TeV}$, while the other components have heavier masses, $\sim 2-5 \mathrm{TeV}$, and hence their contribution to the production and the decay of $\mathcal{S}$ are relatively suppressed.

The SM electroweak symmetry breaking (EWSB) in the left-right model, in general, is achieved by the neutral (diagonal) component of the bidoublet field $\phi(2,2,0,1)$ acquiring a VEV. The fermion content of the model is the same as the SM. There are seven gauge bosons in the model, $W_{L}^{i}, W_{R}^{i}$ (with $i=1,2,3)$, and $W_{B L}$, with the gauge couplings $g_{L}, g_{R}$, and $g_{B L}$, associated with the $\mathrm{SU}(2)_{L}, \mathrm{SU}(2)_{R}$, and $\mathrm{U}(1)_{B-L}$ gauge symmetries, respectively. Using the notation of [21, 23], the symmetry breaking pattern of our model is given by

$$
\operatorname{SO}(10) \underset{\langle\mathbf{2 1 0}\rangle}{\stackrel{M_{U}}{\longrightarrow}} G_{224} \underset{\langle\mathbf{2 1 0}\rangle}{\stackrel{M_{C}}{\longrightarrow}} G_{2213} \underset{\langle\mathbf{1 2 6}\rangle}{\stackrel{M_{R}}{\longrightarrow}} G_{213} \underset{\langle\mathbf{1 0}\rangle}{\stackrel{M_{Z}}{\longrightarrow}} G_{13},
$$

where we assume $M_{R}=5 \mathrm{TeV}$ in our analysis.

In choosing the $\mathrm{SO}(10)$ multiplets for breaking the symmetries (by acquiring appropriate VEVs), we follow the common tradition in the literature as follows. The first stage of the symmetry breaking, where $\mathrm{SO}(10)$ is broken into the Pati-Salam group $G_{224}$, is realized by the singlet $(1,1,1)_{210}$ of 
210. Note that $(1,1,1)_{210}$ is odd under the $D$-parity $[10,11]$, and hence it is broken at this stage as well. Therefore, below the scale $M_{U}$, we have $g_{L} \neq g_{R}$, since they evolve under the influence of different particle contents below this energy scale according to the ESH and the minimal fine-tuning principle. The second stage, where the Pati-Salam group is broken into the left-right group $G_{2213}$, can be accomplished by $(1,1,15)_{210} \equiv \Sigma(1,1,15)$ acquiring a VEV. The breaking of $G_{2213}$ down to the SM gauge group $G_{213}$ is achieved by the $G_{2213}$ multiplet $(1,3,2,1)_{126} \equiv \Delta_{R_{1}}(1,3,2,1)$ which belongs to the Pati-Salam multiplet $(1,3,10)_{126} \equiv \Delta_{R}(1,3,10)$ which is a member of the $\mathrm{SO}(10)$ multiplet 126. In our model, $\Delta_{R_{1}}(1,3,2,1)$ acquires a VEV at around $5 \mathrm{TeV}$ which also set the value of the symmetry breaking scale $M_{R}$. Note that $\Delta_{R_{1}}$ is the regular $\mathrm{SU}(2)_{R}$ triplet usually used in the literature in order to break the $G_{2213}$ symmetry.

\section{Unification of the Couplings}

In this section, we discuss how the unification of the couplings is achieved and derive the values of the symmetry breaking scales. We have only two intermediate scales in our model in between the unification scale $M_{U}$ and the EWSB scale $M_{Z}$, which are $M_{C}$ and $M_{R}$, where the value of $M_{\mathrm{R}}$ is chosen to be $5 \mathrm{TeV}$.

The $\mathrm{TeV}$-scale left-right model with light colored scalars in the minimal nonsupersymmetric SO(10) GUT scheme has recently been discussed in [23]. Here, the situation has a slight difference in one of the components in the decomposition of the left-right multiplet $\Delta_{R_{3}}$ (shown in (1)) into the SM gauge group, which is $\Delta_{R_{3}}^{4 / 3}$ whose mass is $\sim 1 \mathrm{TeV}$. Therefore, the renormalization group (RG) running of the gauge couplings at this energy scale is slightly different. The other particle $\delta$ which, we assume, has a mass also around $\sim 1 \mathrm{TeV}$, does naturally not contribute to the running since it is a SM-singlet.

3.1. Basics. We label the energy intervals in between symmetry breaking scales starting from $\left[M_{Z}, M_{R}\right]$ up to $\left[M_{C}, M_{U}\right]$ with Roman numerals as follows:

$$
\begin{aligned}
& \text { I: } \underbrace{\left[M_{Z}, M_{R}\right]}_{G_{213}} \text {; } \\
& \text { II: } \underbrace{\left[M_{R}, M_{C}\right]}_{G_{2213}} \text {; } \\
& \text { III: } \underbrace{\left[M_{C}, M_{U}\right]}_{G_{224}} \text {. }
\end{aligned}
$$

The boundary/matching conditions we impose on the couplings at the symmetry breaking scales are

$$
\begin{aligned}
& M_{U}: g_{L}\left(M_{U}\right)=g_{R}\left(M_{U}\right)=g_{4}\left(M_{U}\right), \\
& M_{C}: \sqrt{\frac{2}{3}} g_{B L}\left(M_{C}\right)=g_{3}\left(M_{C}\right)=g_{4}\left(M_{C}\right), \\
& M_{R}: \frac{1}{g_{1}^{2}\left(M_{R}\right)}=\frac{1}{g_{R}^{2}\left(M_{R}\right)}+\frac{1}{g_{B L}^{2}\left(M_{R}\right)}, \\
& g_{2}\left(M_{R}\right)=g_{L}\left(M_{R}\right),
\end{aligned}
$$

$$
M_{Z}: \frac{1}{e^{2}\left(M_{Z}\right)}=\frac{1}{g_{1}^{2}\left(M_{Z}\right)}+\frac{1}{g_{2}^{2}\left(M_{Z}\right)}
$$

The low energy data which we will use as boundary conditions to the RG running are $[47,48]$

$$
\begin{aligned}
\alpha & =\frac{1}{127.9} ; \\
\alpha_{s} & =0.118 ; \\
\sin ^{2} \theta_{W} & =0.2312,
\end{aligned}
$$

and all are evaluated at $M_{Z}=91.2 \mathrm{GeV}$, which gives

$$
\begin{aligned}
& g_{1}\left(M_{Z}\right)=0.36, \\
& g_{2}\left(M_{Z}\right)=0.65, \\
& g_{3}\left(M_{Z}\right)=1.22 .
\end{aligned}
$$

Note that the coupling constants are all required to remain in the perturbative regime during the evolution from $M_{U}$ down to $M_{Z}$.

3.2. One-Loop RG Running. For a given particle content; the gauge couplings, in an energy interval $\left[M_{A}, M_{B}\right]$, are evolved according to the one-loop RG relation

$$
\frac{1}{g_{i}^{2}\left(M_{A}\right)}-\frac{1}{g_{i}^{2}\left(M_{B}\right)}=\frac{a_{i}}{8 \pi^{2}} \ln \frac{M_{B}}{M_{A}},
$$

where the RG coefficients $a_{i}$ are given by $[49,50]$ as

$$
\begin{aligned}
a_{i}= & -\frac{11}{3} C_{2}\left(G_{i}\right)+\frac{2}{3} \sum_{R_{f}} T_{i}\left(R_{f}\right) \cdot d_{1}\left(R_{f}\right) \cdots d_{n}\left(R_{f}\right) \\
& +\frac{\eta}{3} \sum_{R_{s}} T_{i}\left(R_{s}\right) \cdot d_{1}\left(R_{s}\right) \cdots d_{n}\left(R_{s}\right) .
\end{aligned}
$$

Here, the two summations are over irreducible chiral representations of fermions $R_{f}$ and those of scalars $R_{s}$. The coefficient $\eta$ is either 1 or $1 / 2$, depending on whether the representation is complex or real, respectively. The quadratic Casimir for the adjoint representation of the group $G_{i}$ is $C_{2}\left(G_{i}\right)$ and $T_{i}$ is the Dynkin index of each representation. For $\mathrm{U}(1)$ group, $C_{2}(G)=0$ and

$$
\sum_{f, s} T=\sum_{f, s}\left(\frac{Y}{2}\right)^{2}
$$

where $Y / 2$ is the $U(1)$ charge, the factor of $1 / 2$ coming from the traditional normalizations of the hypercharge and $B-L$ charges. The $a_{i}$ 's differ depending on the particle content in each energy interval, which changes every time symmetry breaking occurs. We will distinguish the $a_{i}$ 's in different intervals with the corresponding roman numeral superscript, cf. (3). 
TABLE 1: The Higgs content and the RG coefficients in the energy intervals for our model.

\begin{tabular}{lcc}
\hline Interval & Higgs content & RG coefficients \\
\hline III & $\phi(2,2,1), \Delta_{R}(1,3,10), \Sigma(1,1,15)$ & $\left(a_{L}, a_{R}, a_{4}\right)^{\mathrm{III}}=(-3,11 / 3,-7)$ \\
II & $\phi(2,2,0,1), \Delta_{R_{1}}(1,3,2,1), \Delta_{R_{3}}(1,3,2 / 3,3)$ & $\left(a_{L}, a_{R}, a_{B L}, a_{3}\right)^{\mathrm{II}}=(-3,-1 / 3,4,-13 / 2)$ \\
I & $\phi_{2}(2,1,1), \mathcal{S}(1,1,1), \chi(1,8 / 3,3)$ & $\left(a_{1}, a_{2}, a_{3}\right)^{\mathrm{I}}=(155 / 18,-19 / 6,-41 / 6)$ \\
\hline
\end{tabular}

3.3. Results. The scalar content in the energy intervals are

III: $\phi(2,2,1), \Delta_{R}(1,3,10), \Sigma(1,1,15)$,

$$
\begin{gathered}
\text { II: } \phi(2,2,0,1), \Delta_{R_{1}}(1,3,2,1), \Delta_{R_{3}}\left(1,3, \frac{2}{3}, 3\right), \\
\text { I: } \phi_{2}(2,1,1), \mathcal{S}(1,1,1), \Delta_{R_{3}}^{4 / 3}\left(1, \frac{8}{3}, 3\right) .
\end{gathered}
$$

It is common in the literature that another scalar Pati-Salam multiplet, $\widetilde{\Sigma}(2,2,15)$, is included in interval III for a rich Yukawa phenomenology $[14,15]$. In terms of the RG evolution, which is our main focus here, this extra multiplet would not alter the results noticeably, because its effect in the RG equations would appear as a contribution in the term $\left(-5 a_{L}+\right.$ $3 a_{R}+2 a_{4}$ ) (see (14)), which would be very small compared to the rest of the term. Therefore, for the sake of staying minimal, we do not include this multiplet in our set-up.

The values of the RG coefficients for this Higgs content are listed in Table 1 . The relations between symmetry breaking scales, which can be derived by using the one-loop running equations and the boundary/matching conditions, can be obtained as (for derivation see [21, 23])

$$
\begin{aligned}
2 \pi\left[\frac{3}{\alpha}-\frac{8}{\alpha_{s}}\right]= & \left(3 a_{L}+3 a_{R}-6 a_{4}\right)^{\mathrm{III}} \ln \frac{M_{U}}{M_{C}} \\
& +\left(3 a_{L}+3 a_{R}+3 a_{B L}-8 a_{3}\right)^{\mathrm{II}} \ln \frac{M_{C}}{M_{R}} \\
& +\left(3 a_{1}+3 a_{2}-8 a_{3}\right)^{\mathrm{I}} \ln \frac{M_{R}}{M_{Z}} \\
2 \pi\left[\frac{3-8 s_{w}^{2}}{\alpha}\right]= & \left(-5 a_{L}+3 a_{R}+2 a_{4}\right)^{\mathrm{III}} \ln \frac{M_{U}}{M_{C}} \\
& +\left(-5 a_{L}+3 a_{R}+3 a_{B L}\right)^{\mathrm{II}} \ln \frac{M_{C}}{M_{R}} \\
& +\left(3 a_{1}-5 a_{2}\right)^{\mathrm{I}} \ln \frac{M_{R}}{M_{Z}},
\end{aligned}
$$

where $s_{w} \equiv \sin \theta_{W}$. Using these equations and the experimentally measured quantities in (8) and demanding $M_{R}=5 \mathrm{TeV}$, we obtain the following values:

$$
\begin{aligned}
& M_{C}=10^{15.0} \mathrm{GeV}, \\
& M_{U}=10^{17.9} \mathrm{GeV} .
\end{aligned}
$$

The value for the scale $M_{C}$ is sufficiently high to ensure that the effects induced by the presence of scalar and vectorleptoquarks are suppressed adequately enough to remain consistent with the experimental constraints [51]. Besides, the unification scale $M_{U}$ is high enough to escape the bound on the proton decay induced by gauge boson exchanging operators. We should also note that we have light colortriplets in our model, and as well known they lead to scalar-induced dimension- 6 operators that contribute to the proton decay amplitude. Although these contributions are typically suppressed by small Yukawa couplings, the colortriplets being as light as the $\mathrm{TeV}$-scale can cause a potentially dangerous situation [52]. In such a case, a mechanism is required to adequately suppress these interactions, such as the ones proposed in $[53,54]$.

The value of the unified gauge coupling can be found via the following equation:

$$
\frac{2 \pi}{\alpha_{s}}-\frac{2 \pi}{\alpha_{U}}=a_{4}^{\mathrm{III}} \ln \frac{M_{U}}{M_{C}}+a_{3}^{\mathrm{II}} \ln \frac{M_{C}}{M_{R}}+a_{3}^{\mathrm{I}} \ln \frac{M_{R}}{M_{Z}}
$$

as $\alpha_{U}^{-1} \simeq 47.2$. The running of the couplings is given in Figure 1. Similarly, the gauge couplings at $M_{R}=5 \mathrm{TeV}$ are obtained as

$$
\begin{aligned}
g_{R} & \simeq 0.50 ; \\
g_{L} & \simeq 0.63 ; \\
g_{B L} & \simeq 0.55 ; \\
g_{3} & \simeq 0.99,
\end{aligned}
$$

which, together with the values of the symmetry breaking scales in (16), are the main predictions of the model. Notice that the value of $g_{R}(5 \mathrm{TeV})$ is different from the value of $g_{2}(5 \mathrm{TeV})=g_{L}(5 \mathrm{TeV}) \simeq 0.63$, which is expected due to the fact that the $D$-parity invariance is broken together with the $\mathrm{SO}(10)$ symmetry; hence $g_{R} \neq g_{L}$ below the unification scale $M_{U}$, as mentioned previously. The model also predicts the existence of TeV-scale gauge bosons $W_{R}$ and $Z_{R}$ whose masses at $M_{R}$ are given as

$$
\begin{aligned}
& M_{W_{R}} \approx g_{R} v_{R} ; \\
& M_{Z_{R}} \approx \sqrt{2\left(g_{R}^{2}+g_{B L}^{2}\right)} v_{R},
\end{aligned}
$$

where we choose $v_{R} \equiv M_{R}=\left\langle\Delta_{R_{1}}^{0} \equiv \mathcal{S}\right\rangle=5 \mathrm{TeV}$ which, together with (18), yields

$$
\begin{aligned}
& M_{W_{R}}\left(M_{R}\right) \approx 2.5 \mathrm{TeV}, \\
& M_{Z_{R}}\left(M_{R}\right) \approx 5.3 \mathrm{TeV} .
\end{aligned}
$$

These are the specific predictions of our model. However, we note that $M_{W_{R}}$ and $M_{Z_{R}}$ change significantly with the choice 


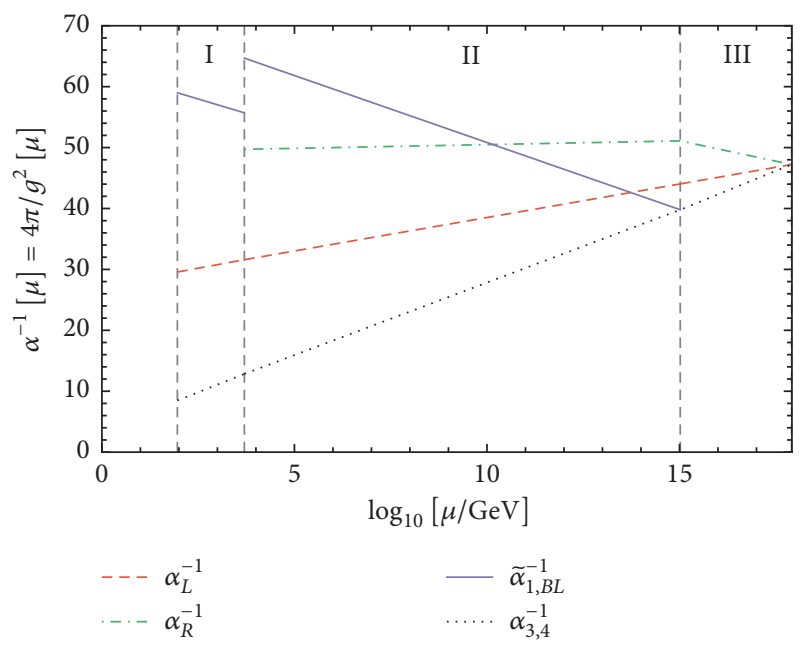

FIGURE 1: Running of the gauge couplings for the model. The vertical dotted lines from left to right correspond to the symmetry breaking scales $M_{Z}, M_{R}$, and $M_{C}$, which also indicate the beginning of the energy intervals I, II, and III, respectively. For $\alpha_{1}^{-1}$ and $\alpha_{B L}^{-1}$, we plot the redefined quantities $\widetilde{\alpha}_{1}^{-1} \equiv(3 / 5) \alpha_{1}^{-1}$ and $\widetilde{\alpha}_{B L}^{-1} \equiv(3 / 2) \alpha_{B L}^{-1}$. Note that the discontinuity on the $\tilde{\alpha}_{1, B L}^{-1}$ plot at the energy scale $M_{R}$ occurs due to the boundary condition given in (6).

of the symmetry breaking scale $M_{R}$. Therefore, these mass values are not very distinctive predictions of the model. The more reliable and robust prediction is rather the values of the gauge couplings in the TeV-scale, given in (18), which do not change noticeably with the choice of the value of $M_{R}$ due to their logarithmic dependence on the energy scale.

Recall that our model is just the left-right model augmented by a colored scalar at the TeV-scale. Therefore, similar to the usual left-right model it allows the right-handed neutrino $N_{R}$ to be Majorana in character. Although there is no mechanism that constrains right-handed neutrino mass $M_{N_{R}}$ in the left-right models, there exist bounds obtained from various low energy processes [55]. The LHC implications of $\mathrm{TeV}$-scale left-right models regarding a heavy Majorana right-handed neutrino for variety of mass ranges have been studied in the literature $[56,57]$. As for the future runs of the LHC; as recently studied in [58], for $g_{R} / g_{L} \sim 0.79$ (which is the case in our model as can be seen in (18)), the $14 \mathrm{TeV}$ LHC searches can probe the range $M_{W_{R}} \lesssim 6.3-7 \mathrm{TeV}$ for $M_{N_{R}}=$ $100-700 \mathrm{GeV}$.

\section{Phenomenology}

In Section 2, we have discussed that the SM-singlet $\delta$ can be as light as $\sim 1 \mathrm{TeV}$ and can potentially be observed at the LHC. Since $\mathcal{S}$ is a SM-singlet, it can not directly couple to the SM fermions and gauge bosons through any dimension4 operator due to gauge invariance. Therefore, in order to produce $\mathcal{S}$ at the LHC, it is necessary to introduce extra colored particles that present in the loop. Similarly, for its decay to pair of EW gauge bosons, we need particles in the loop with nonzero hypercharge. These particles can be scalar, vector, or fermionic in nature. As mentioned previously, we

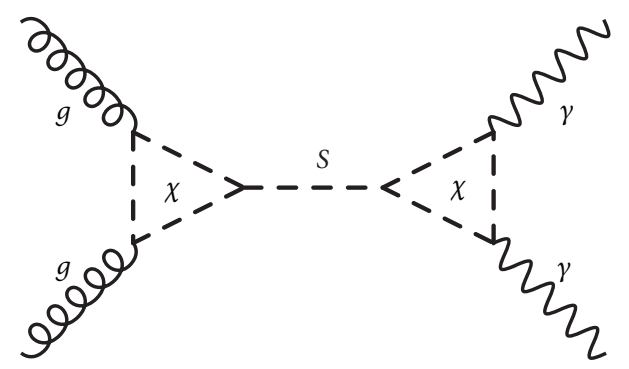

FIGURE 2: The Feynman diagram of the production and decay of $\mathcal{S}$ at the LHC through $\chi$ in the loop.

would like to keep the matter and gauge sectors minimal and want to do a simplistic phenomenological study of that scenario. We, therefore, choose only one colored and hypercharged scalar $\chi$ that appears naturally in our model and can serve both purposes, production and decay of $\mathcal{S}$ through loop interactions. Note that the EM charge of $\chi$ is $4 / 3$ which is the largest among the $\mathrm{TeV}$-scale colored scalars in our model. Therefore, it couples to photon with a relatively greater strength which implies large BR of $\delta$ to diphoton. We further assume that $\chi$ is the lightest among all the colored and EM charged scalars of our model and contributes most in our analysis. We neglect any small contamination from other particles in the loop assuming that they are heavier and thus their effects are relatively suppressed. In Figure 2, we present the Feynman diagram of the production of $\delta$ from gluon-gluon fusion and its decay to two photons through $\chi$ in the loop.

4.1. Production and Decay. The scalar $\delta$ being singlet in nature, there is no tree level couplings of $\delta$ to the SM fermions and gauge bosons. It can decay to a pair of SM gauge bosons only through nonrenormalizable dimension-5 operators. In the potential of the model, there could be some interaction terms which connect $\delta$ with the SM Higgs doublet, which can lead to a mixing between $\mathcal{\delta}$ and the SM Higgs, after EWSB. Consequently, $\mathcal{S}$ can decay to a pair of the SM particles at the tree level. We know from experiments that the $125 \mathrm{GeV}$ scalar observed at the LHC is very much the SM-like Higgs and therefore its mixing with $\mathcal{S}$ is expected to be small. For simplicity, we consider the $\delta$ - $h$ mixing, and therefore the partial widths of $\delta$ to two SM fermions or two Higgs bosons are negligible. Since $\chi$ carries color and hypercharge, it couples to the gluon and the $B_{\mu}$ (hypercharge) fields. Note that there is no coupling between $\chi$ and $W$ bosons, since $\chi$ is a singlet under $\mathrm{SU}(2)_{L}$. In the effective Lagrangian, we have the following dimension-5 operators for the interactions of $\mathcal{S}$ with the SM gauge bosons prior to EWSB.

$$
\mathscr{L} \supset-\frac{1}{4} \kappa_{g} \delta G_{\mu \nu}^{a} G^{a \mu \nu}-\frac{1}{4} \kappa_{B} \delta B_{\mu \nu} B^{\mu \nu},
$$

where $G_{\mu \nu}^{a}$ and $B_{\mu \nu}$ are the field-strength tensors for $\mathrm{SU}(3)_{c}$ and $\mathrm{U}(1)_{Y}$ gauge groups, respectively. Effective couplings $\kappa_{g}$ and $\kappa_{B}$ are associated with the gluon and the $B_{\mu}$ fields, respectively. These couplings can be computed from the knowledge of the trilinear coupling related to the $\delta\left|\chi_{i}\right|^{2}$ interaction term. In general, for $N_{f}$ number of colored scalars 
$\chi_{i}$ with hypercharge $Y_{i}$ and for an interaction term $y_{\mathcal{S}}^{i} \mathcal{S}\left|\chi_{i}\right|^{2}$, the effective couplings are expressed as

$$
\begin{aligned}
& \kappa_{g}=\frac{\alpha_{S}}{2 \pi}\left|\sum_{i=1}^{N_{f}} \frac{1}{6} C_{R}^{i} \frac{y_{\mathcal{S}}^{i}}{M_{\chi_{i}}^{2}} I_{0}\left(\frac{4 M_{\chi_{i}}^{2}}{M_{\mathcal{S}}^{2}}\right)\right|, \\
& \kappa_{B}=\frac{\alpha}{2 \pi c_{W}^{2}}\left|\sum_{i=1}^{N_{f}} \frac{1}{6} d_{R}^{i}\left(\frac{Y_{i}}{2}\right)^{2} \frac{y_{\mathcal{S}}^{i}}{M_{\chi_{i}}^{2}} I_{0}\left(\frac{4 M_{\chi_{i}}^{2}}{M_{\mathcal{S}}^{2}}\right)\right|,
\end{aligned}
$$

where $d_{R}^{i}$ is the dimension of the $\mathrm{SU}(3)$ representation (e.g., $d_{R}=3$ for triplet and $d_{R}=8$ for octet representations) and $C_{R}^{i}$ is the index of the $\mathrm{SU}(3)$ representation (e.g., $C_{R}=1 / 2$ for triplet and $C_{R}=3$ for octet representations). The strong and the electromagnetic couplings are denoted by $\alpha_{S}$ and $\alpha$, respectively. The cosine of the Weinberg angle is denoted as $c_{W}$. The loop function $I_{0}$ is given by

$$
I_{0}(\tau)=-3 \tau\left[1-\tau\left\{\sin ^{-1}\left(\frac{1}{\sqrt{\tau}}\right)\right\}^{2}\right]
$$

For only one colored triplet and hypercharged $(Y=8 / 3)$ scalar $\chi, N_{f}=1, d_{R}=3$, and $C_{R}=1 / 2$. To keep our results as model independent as possible, we assume $y_{\mathcal{S}}=\kappa \Lambda$, where $\Lambda$ is some new physics scale (this can be chosen as $M_{R}$ ) for which we choose $5 \mathrm{TeV}$ for all our computations and we keep $\kappa$ as a free parameter. The BRs of $\delta$ to $g g, \gamma \gamma, Z \gamma$, and $Z Z$ modes are $90.6 \%, 5.6 \%, 3.3 \%$, and $0.5 \%$ respectively.

Here, we assume that $M_{\chi}>M_{\mathcal{S}} / 2$, and therefore $\delta$ cannot decay to a $\chi$ pair. It is important to note that the BR depends only on $Y$, not on the other parameters. This is because all the partial widths, and hence the total width, scale as $\kappa^{2} \Lambda^{2}$ and the loop function $I_{0}$ (for any values of $M_{\mathcal{S}}$ and $M_{\chi}$ ) would be the same for all the partial widths. The scalar $\mathcal{S}$ has the largest BR in the dijet channel. We expect the BR in the $\gamma \gamma, Z \gamma$, and $Z Z$ are of similar order, but $Z Z$ mode is suppressed due to its phase space factor. The total width $\Gamma_{\mathcal{S}}$ is a function of $M_{\mathcal{S}}, M_{\chi}$, and $\kappa \Lambda$. In Figure 3, we show $\Gamma_{\mathcal{S}}$ as functions of $M_{\chi}$ for $M_{\mathcal{S}}=1 \mathrm{TeV}$ for three different values of $\kappa$ assuming $\Lambda=5 \mathrm{TeV}$. As mentioned previously, $\Gamma_{\mathcal{S}}$ scales as $\kappa^{2} \Lambda^{2}$ and one can easily estimate the total width for other values of $\kappa \Lambda$ from this plot.

4.2. Exclusions from LHC Data. To derive bounds on the model parameters from the LHC data and related numerical analysis, we implement the Lagrangian given in (21) in FeynRules2.0 [59] to generate the model files for the MAdGraph5 [60] event generator. We use CTEQ6L1 [61] parton distribution functions (PDF) to compute cross sections. We fix the factorization and renormalization scales at $M_{\mathcal{S}}$ for all our numerical computations.

For our phenomenological analysis, we have only three free parameters, namely, $M_{\mathcal{S}}, M_{\chi}$, and $\kappa$ (we choose $\Lambda=$ $5 \mathrm{TeV}$ for all our numerical computations). We first derive bounds on the parameters from the latest LHC $13 \mathrm{TeV} \gamma \gamma$ $[62,63], Z \gamma[64,65], Z Z[66]$, and $j j[67,68]$ resonance search data. The observed upper limit (UL) at $95 \%$ confidence

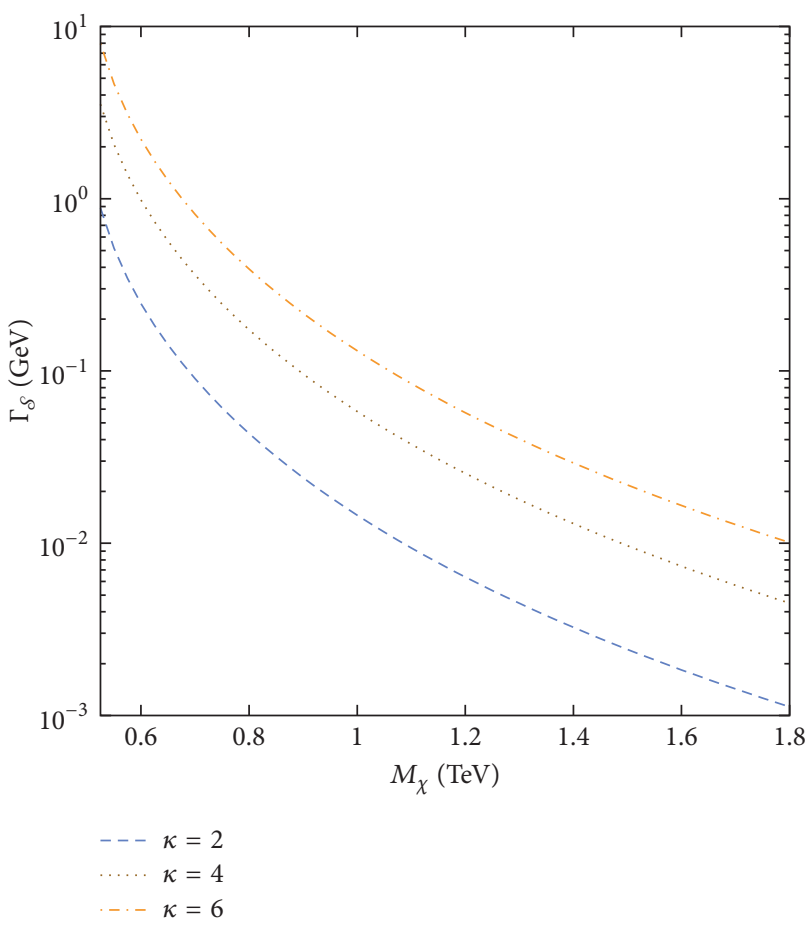

FIgURE 3: Total width of $\mathcal{S}$ for $M_{\mathcal{\delta}}=1 \mathrm{TeV}$ as functions of $M_{\chi}$ for $\kappa=2,4,6$ assuming $\Lambda=5 \mathrm{TeV}$.

level (CL) on the cross sections for the resonance mass of $1 \mathrm{TeV}$ of four type of resonances is given by

$$
\begin{aligned}
\sigma_{\gamma \gamma} & \lesssim 1 \mathrm{fb}, \\
\sigma_{Z \gamma} & \lesssim 10 \mathrm{fb}, \\
\sigma_{Z Z} & \lesssim 20 \mathrm{fb}, \\
\sigma_{j j} & \leqslant 7.5 \mathrm{pb} .
\end{aligned}
$$

These values are used in Figure 4(a) where we show the excluded parameter space (colored regions) in $M_{\chi}-\kappa$ plane for $M_{\mathcal{S}}=1 \mathrm{TeV}$. The excluded regions shown in orange, green, blue, and brown are derived from the $\gamma \gamma, Z \gamma, Z Z$, and $j j$ resonance search data. We can see that the diphoton data is the most powerful in constraining the parameter space in $M_{\chi}-\kappa$ plane. In Figure 4(b), we present the excluded regions in $M_{\mathcal{S}}-M_{\chi}$ plane for different $\kappa$ with $\Lambda=5 \mathrm{TeV}$ from the latest $13 \mathrm{TeV}$ combined ATLAS and CMS diphoton resonance search data. Cross section ULs $\left(\sigma_{i}\right)$ from different experiments and the corresponding uncertainties $\left(\Delta \sigma_{i}\right)$ are combined statistically using the following relations:

$$
\begin{aligned}
& \frac{1}{\left(\Delta \sigma_{c}\right)^{2}}=\sum_{i} \frac{1}{\left(\Delta \sigma_{i}\right)^{2}} \\
& \frac{\sigma_{c}}{\left(\Delta \sigma_{c}\right)^{2}}=\sum_{i} \frac{\sigma_{i}}{\left(\Delta \sigma_{i}\right)^{2}},
\end{aligned}
$$

where $\sigma_{c}$ is the combined cross section and $\Delta \sigma_{c}$ is the uncertainty associated with it. In case of asymmetric uncertainties, 


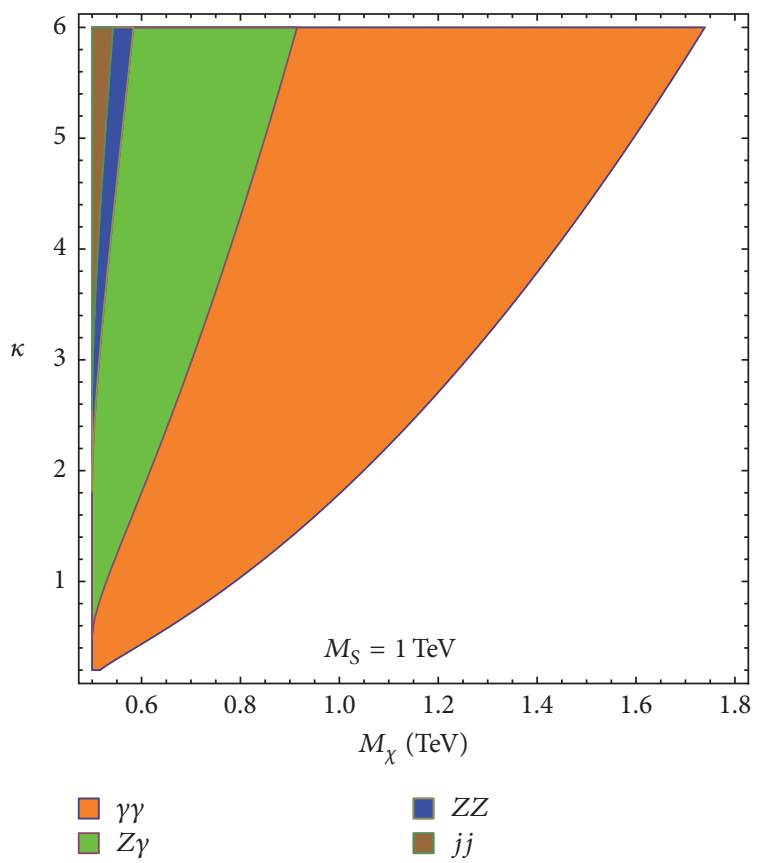

(a)

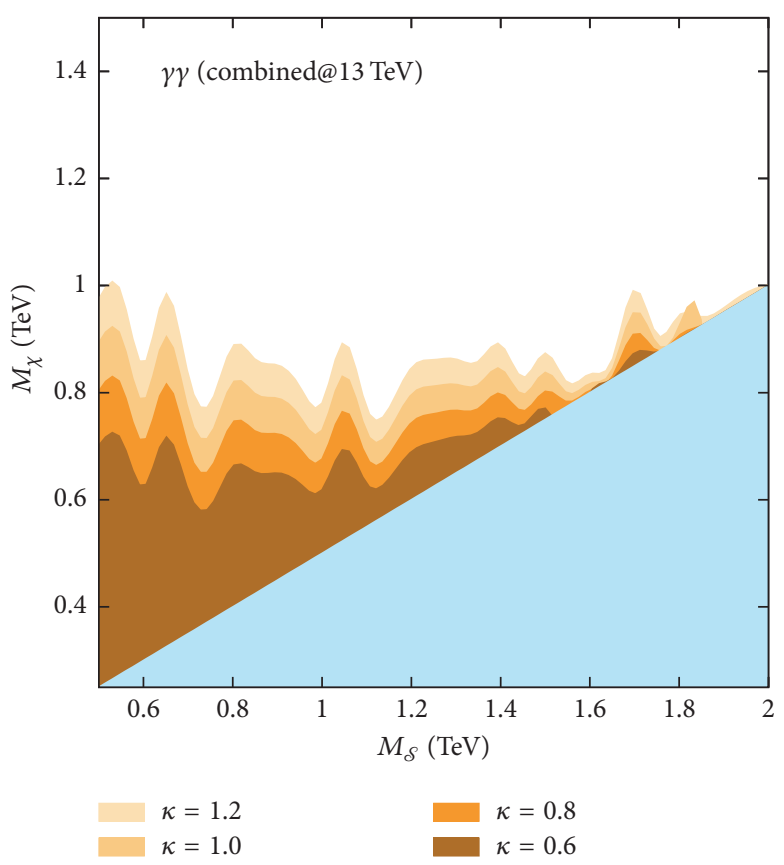

(b)

Figure 4: (a) The excluded region in $M_{\chi}-\kappa$ plane for $M_{\mathcal{S}}=1 \mathrm{TeV}$ from the $13 \mathrm{TeV}$ LHC data. The orange, green, blue, and brown regions represent the region ruled out by the $\gamma \gamma, Z \gamma, Z Z$, and $j j$ resonance search data, respectively. (b) The excluded region in $M_{\mathcal{S}}-M_{\chi}$ plane for different $\kappa$ with $\Lambda=5 \mathrm{TeV}$ using the $13 \mathrm{TeV}$ combined ATLAS and CMS diphoton resonance search. ATLAS and CMS data are combined statistically using (25). The widths of the resonance assumed by ATLAS and CMS are $4 \mathrm{MeV}$ [62] and $140 \mathrm{MeV}$ [63]. The sky-blue region cannot be probed in our analysis as we assume $M_{\chi}>M_{\delta} / 2$.

we get $\Delta \sigma_{i}$ by averaging upper and lower uncertainties. Although uncertainties are used to compute $\sigma_{c}$, we have not shown the uncertainty bands in the exclusion plots for simplicity. The sky-blue regions in these plots cannot be probed in our set-up as we always assume $M_{\chi}>M_{\mathcal{S}} / 2$. If $M_{\chi}<$ $M_{\mathcal{S}} / 2$, the $\mathcal{S} \rightarrow \chi \chi$ decay becomes kinematically allowed and becomes the dominant decay mode of $\mathcal{S}$. This will make the diphoton and other branching modes suppressed. Therefore, observing $\delta$ in the $\gamma \gamma, Z \gamma, Z Z$, and $j j$ resonance searches becomes much more challenging. One should note that exclusion regions are not very sensitive to the $M_{\chi}$ values for a fixed $\kappa$. This is because, for heavier resonances, the cross section ULs are not very sensitive to the resonance mass due to lack of statistics and therefore the quantity $\sigma\left(M_{\mathcal{S}}, M_{\chi}\right) \times$ $\mathrm{BR}$ should remain insensitive for heavier resonances. The reduction in the production cross section as we increase $M_{\mathcal{S}}$ is compensated by the slight change in $M_{\chi}$ since $\sigma\left(M_{\mathcal{S}}, M_{\chi}\right)$ quantity is very sensitive to the $M_{\chi}$. Note that these bounds are derived from the observed 95\% CL UL on the cross sections. Consideration of uncertainties on the cross sections limits would relax the derived bound somewhat. In all our computations, we have considered a next-to-leading order $K$ factor of 2 to account for the higher-order effects [69].

4.3. Future Prospects at the LHC. In this subsection, we look at the prospect of discovering $\delta$ at the $13 \mathrm{TeV}$ LHC runs with high integrated luminosities. In previous subsection, we find that the most stringent bounds come from the diphoton data. Therefore, we only focus on the diphoton final state for the present prospect study. After event generation, we use Pythia6 [70] for parton shower and hadronization. The subsequent detector simulation is done using DeLPHes3 [71] package. Jets are clustered with FASTJET [72] using the anti$k_{T}$ algorithm [73] with the clustering parameter, $R=0.4$. We use TMVA [74] for the multivariate analysis.

Signal events are generated with up to two jets, that is, $p p \rightarrow \delta(\rightarrow \gamma \gamma)+0,1,2$ jets which are MLM [75] merged at a matching scale $Q_{\text {cut }}=50 \mathrm{GeV}$. The dominant (roughly $90 \%$ ) SM background for this signal comes from the $q q \rightarrow \gamma \gamma$ process. Similar to the signal, we generate this background by merging $p p \rightarrow \gamma \gamma+0,1,2$ jets processes at $Q_{\text {cut }}=15 \mathrm{GeV}$. We only consider this dominant background in our analysis. Appropriate matching scales for signal and background are determined by assuring smooth transition in the differential jet-rate distributions between events with $N$ and $N+1$ jets and matched cross sections are within $\sim 10 \%$ of the zero jet contribution. We also check the stability of the matched cross section with the variation of $Q_{\text {cut }}$ once it is properly chosen.

The $13 \mathrm{TeV}$ diphoton data already set an UL on $\sigma \times \mathrm{BR} \sim$ $1 \mathrm{fb}$ for the resonance mass of around $1 \mathrm{TeV}$. Therefore, it is very challenging to observe such a signal over the large SM background. ATLAS and CMS collaborations use cut-based technique in their diphoton resonance searches at the $13 \mathrm{TeV}$ LHC. In this paper, to obtain better sensitivity, we use a MVA to discriminate tiny signal from the large SM background. ATLAS and CMS ULs on $\sigma \times$ BR slightly depend on the width 
TABLE 2: Input variables used for MVA to separate the signal from the background and their relative importance (RI). These numbers are shown for $M_{\mathcal{\delta}}=1 \mathrm{TeV}$.

\begin{tabular}{lc}
\hline Variables & $\mathrm{RI} \times 10^{-1}$ \\
\hline$p_{T}\left(\gamma_{1}\right)$ & 1.22 \\
$p_{T}\left(\gamma_{2}\right)$ & 1.31 \\
$\eta\left(\gamma_{2}\right)$ & 1.02 \\
$\eta\left(\gamma_{1}\right)$ & 1.11 \\
$\Delta R\left(\gamma_{1}, \gamma_{2}\right)$ & 1.30 \\
$M\left(\gamma_{1}, \gamma_{2}\right)$ & 3.25 \\
$N_{\text {jet }}$ & 0.78 \\
\hline
\end{tabular}

of the resonance but we use a fixed width of $1 \mathrm{GeV}$ for all $M_{\delta}$ in the following analysis for simplicity. The width of $\mathcal{S}$ is a function of model parameters, namely, $M_{\delta}, M_{\chi}$, and $\kappa \Lambda$. Instead of choosing a specific benchmark, we use $\Gamma_{\mathcal{S}}=$ $1 \mathrm{GeV}$ for our MVA. This analysis is insensitive to the actual width choice as long as $\Gamma_{\mathcal{\delta}} \ll M_{\delta}$; that is, the narrow width approximation is well-valid.

We generate signal and background events with some basic transverse momentum $\left(p_{T}\right)$, pseudorapidity $(\eta)$ and separation in $\eta-\phi$ plane $(\Delta R)$ cuts as follows:

$$
\begin{aligned}
p_{T}(x) & >25 \mathrm{GeV}, \\
|\eta(x)| & <2.5, \\
\Delta R(x, y) & >0.4,
\end{aligned}
$$

where $x, y=\{\gamma, j\}$. We use a strong selection cut on the invariant mass of the photon pair, $\left|M(\gamma \gamma)-M_{\delta}\right|<100 \mathrm{GeV}$ to reduce the huge diphoton background before passing events to TMVA. For MVA, we use the Boosted Decision Tree (BDT) algorithm where we feed the following seven kinematic variables: $p_{T}\left(\gamma_{1}\right), p_{T}\left(\gamma_{2}\right),\left|\eta\left(\gamma_{1}\right)\right|,\left|\eta\left(\gamma_{2}\right)\right|, \Delta R\left(\gamma_{1}, \gamma_{2}\right), M\left(\gamma_{1}, \gamma_{2}\right)$, and jet multiplicity ( $\gamma_{1}$ and $\gamma_{2}$ are the two selected photons ordered according to their $p_{T}$ ). In Figure 5, we show the signal (blue) and background (red) distributions of these variables used in MVA. We choose these simple variables which are less correlated and have sufficiently good discriminating power. In Table 2, we show the relative importance (RI) of these variables for the benchmark mass $M_{\delta}=1 \mathrm{TeV}$. We find that the two variables $M\left(\gamma_{1}, \gamma_{2}\right)$ and $\Delta R\left(\gamma_{1}, \gamma_{2}\right)$ are very effective in discriminating signal from background. Other variables like $p_{T}$ and $\eta$ of photons also have reasonably good discriminating power. We obtain the cut efficiency of almost $75 \%$ for the signal but as small as $10 \%$ for the background for the whole range of $M_{\mathcal{S}}$ we considered. It is important to mention that this set of seven variables used might not be the optimal one. There is always a scope to improve the analysis with cleverer choices of variables.

The $\mathrm{BDT}$ algorithm is prone to overtraining and therefore one should always be careful while using it in MVA. Overtraining of the signal and background test samples can usually happen due to the improper choices of BDT tuning parameters. Whether a test sample is overtrained or not can be checked by using the Kolmogorov-Smirnov (KS) statistics. Generally, a test sample is not overtrained if the corresponding KS probability lies within the range 0.1 to 0.9 . In our analysis, we use two statistically independent samples for each $M_{\delta}$ choice, one for training and the other for testing the BDT. In Figure 6(a), we show the BDT response of the signal and background for the benchmark mass $M_{\delta}=1 \mathrm{TeV}$. From the BDT response, one can see that a BDT cut around $\sim 0$ can effectively separate the signal from the background and lead to best significance. In Figure 6(b), we show the $M\left(\gamma_{1}, \gamma_{2}\right)$ distributions for the signal and the background for $M_{S}=1 \mathrm{TeV}$ at the $13 \mathrm{TeV}$ LHC with $\mathscr{L}=300 \mathrm{fb}^{-1}$. This plot is shown for the significance of $5 \sigma$ where $\sigma=N_{S} / \sqrt{N_{S}+N_{B}}$ and the numbers of signal and background events that survive after the optimal BDT cut $(>0)$ are $N_{S}=69$ with cut efficiency 0.75 and $N_{B}=120$ with cut efficiency 0.1 , respectively.

In Figures $7(\mathrm{a})$ and $7(\mathrm{~b})$, we show the $5 \sigma$ discovery contours in $M_{\mathcal{S}}-M_{\chi}$ plane for different $\kappa$ at the $13 \mathrm{TeV}$ LHC for 100 and $300 \mathrm{fb}^{-1}$ integrated luminosities, respectively. As stated earlier, the sky-blue region, that is, $M_{\chi}<M_{\delta} / 2$, is not considered in our analysis. We observe that the discovery reach for $100 \mathrm{fb}^{-1}$ run in Figure 7(a) is not much improved from the bounds obtained in Figure 4. But for $300 \mathrm{fb}^{-1}$ run, a substantially bigger region of parameter space can be probed. In Figure 7(c), we show the expected 95\% CL exclusion plot in $M_{\delta}-M_{\chi}$ plane for $\mathscr{L}=300 \mathrm{fb}^{-1}$. It is obvious that the parameter space which can be excluded with $95 \% \mathrm{CL}$ is much bigger than the parameter space which can be discovered with $5 \sigma$ significance. As previously mentioned, the limits on $\sigma \times \mathrm{BR}$ for scalar decay to diphoton are already very strict. Therefore, to observe such a scalar at the LHC is very challenging and we need a more dedicated analysis for that.

In this paper, we choose to use a MVA for the LHC prospect study to achieve better sensitivity to the parameter space compared to a cut-based analysis. To give the readers a rough idea of gain in sensitivity, we wish to present here a quantitative comparison between the two types of analyses for the benchmark mass $M_{\delta}=1 \mathrm{TeV}$. We apply further the following hard cuts on photons, namely, $p_{T}\left(\gamma_{1}\right), p_{T}\left(\gamma_{2}\right)>$ $200 \mathrm{GeV}$ and $\left|M\left(\gamma_{1}, \gamma_{2}\right)-M_{\delta}\right|<50 \mathrm{GeV}$ on the events that are used for the BDT analysis. In context of Figure 6(b), we have discussed previously that the numbers of signal and background events which survive after the optimal BDT cut (around $\sim 0$ ) are 69 and 120, respectively. The corresponding signal and background events that survive after the cut-based analysis are 65 and 432 , respectively, which leads to a $\sim 3 \sigma$ significance. One can see, therefore, that BDT analysis is very effective in terms of background reduction compared to a cut-based analysis. Note that this set of cuts is not fully optimized (but fairly good) and one can vary these cuts to find the optimized set of cuts to improve the significance from $\sim 3 \sigma$. But an optimized BDT analysis is always expected to perform better than an optimized cut-based analysis as long as a clever set of variables are used. A BDT analysis is usually more effective than a cut-based analysis especially in the low mass (here low $M_{\delta}$ ) region. For heavier masses, where the SM background is expected to be very small compared to the signal, an optimized cut-based analysis can compete to an optimized BDT analysis. 

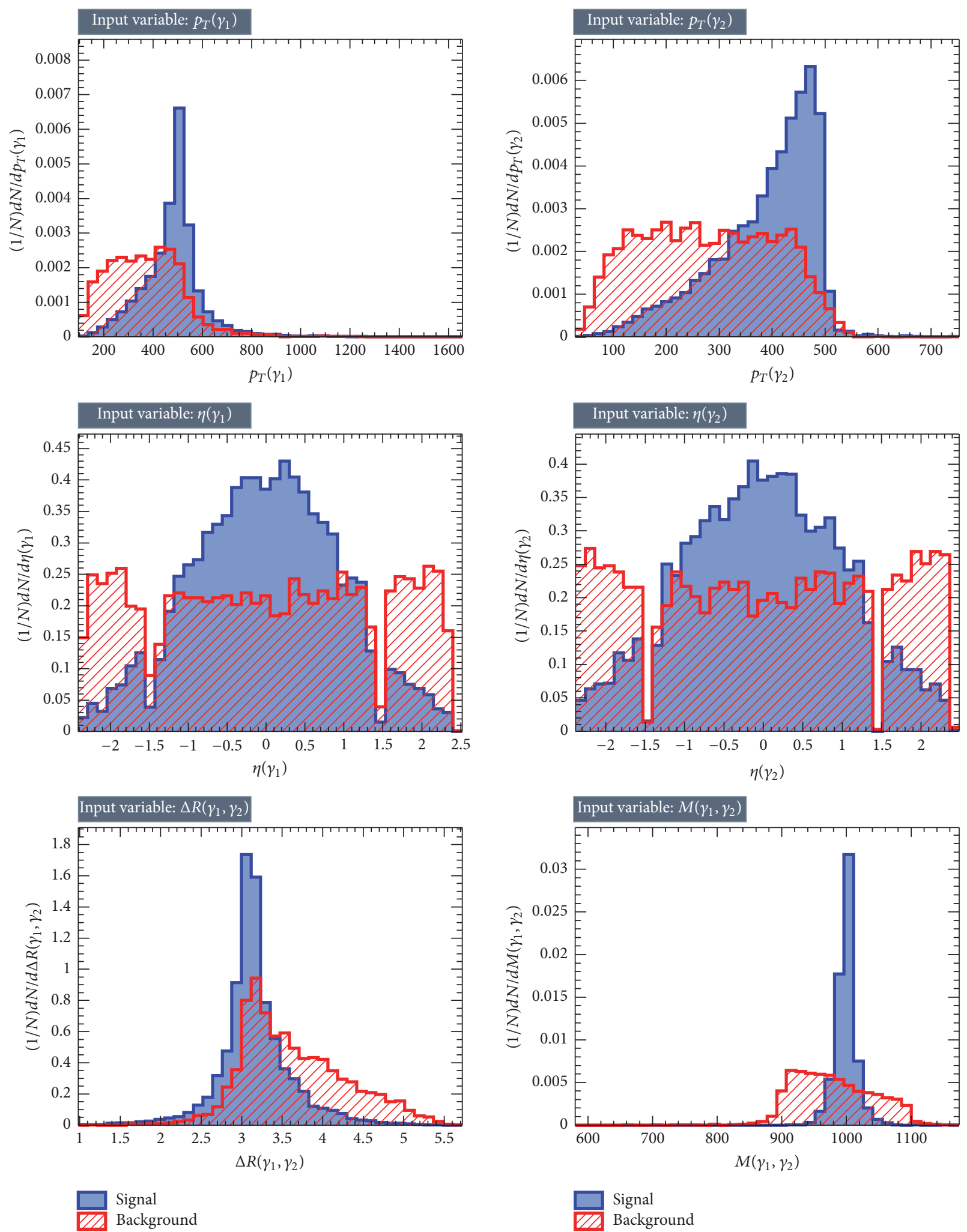

FIGURE 5: Signal (blue) and background (red) distributions of the input variables used for MVA. These distributions are generated for $M_{\delta}=$ $1 \mathrm{TeV}$ assuming $\Gamma_{\delta}=1 \mathrm{GeV}$. We do not present the jet multiplicity distribution here since it has the smallest RI in the MVA as shown in Table 2 and therefore the $N_{\text {jet }}$ distribution would not differ much for the signal and the background. 


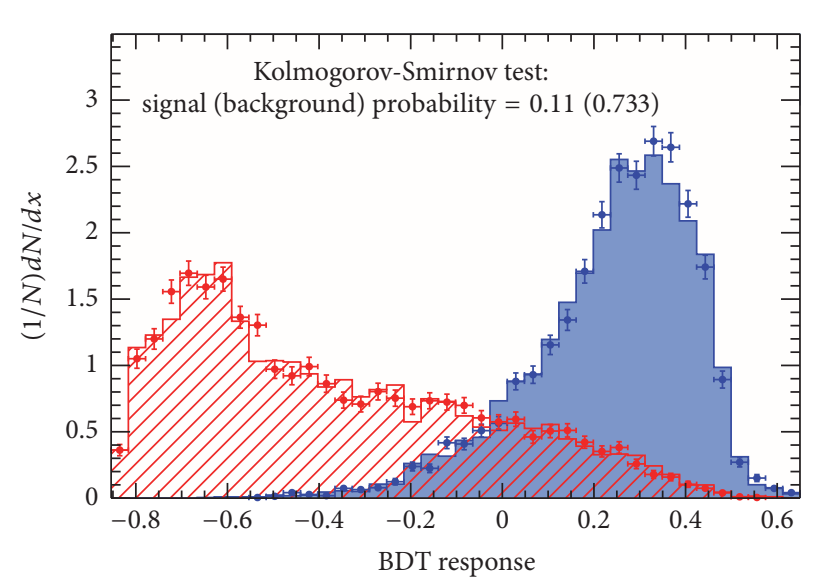

Signal (test sample)

Background (test sample)

- Signal (training sample)

- Background (training sample)

(a)

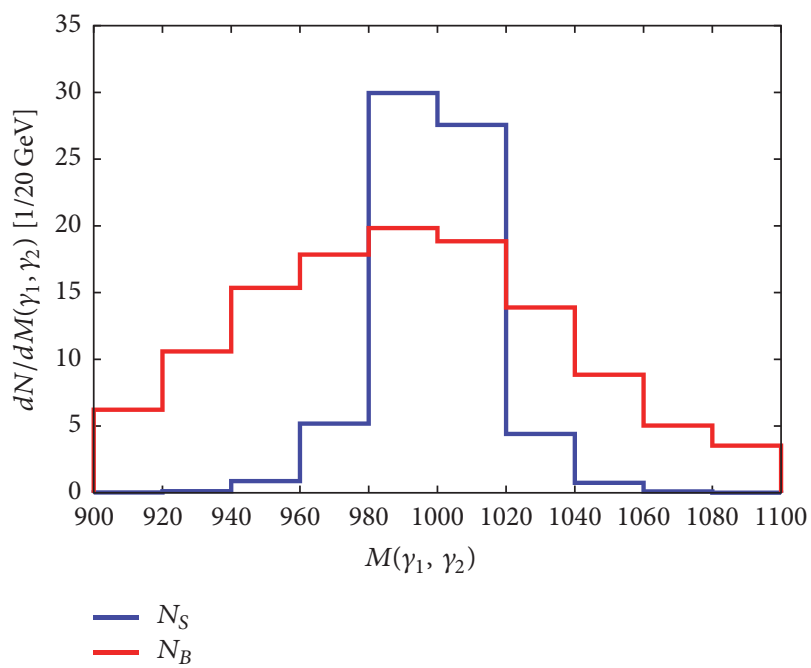

(b)

Figure 6: (a) BDT response of the signal (blue) and the background (red) for $M_{s}=1 \mathrm{TeV}$. (b) $M\left(\gamma_{1}, \gamma_{2}\right)$ distributions for the signal (blue) and the background (red) for $M_{\mathcal{S}}=1 \mathrm{TeV}$ after applying the optimal BDT cut at $\sim 0$ to obtain $5 \sigma$ significance at the $13 \mathrm{TeV} \mathrm{LHC}_{\mathrm{Hith}} \mathscr{L}=300 \mathrm{fb}{ }^{-1}$.

\section{Summary}

In this paper, we explore the phenomenology of TeV-scale scalars in the nonsupersymmetric $\mathrm{SO}(10)$ grand unification framework. In particular, we investigate the LHC phenomenology of a SM-singlet scalar $\mathcal{S}$ which interacts with gluons and photons through loop interactions with a colortriplet hypercharged scalar $\chi$ which is remnant from the breaking of the Pati-Salam gauge group, $\mathrm{SU}(2)_{L} \otimes \mathrm{SU}(2)_{R} \otimes$ $\mathrm{SU}(4)_{C}$. The part of the model that lies in the TeV-scale is the left-right model, whose gauge group is $\mathrm{SU}(2)_{L} \otimes \mathrm{SU}(2)_{R} \otimes$ $\mathrm{U}(1)_{B-L} \otimes \mathrm{SU}(3)_{C}$, augmented with the color-triplet scalar $\chi$. The scalar $\mathcal{S}$ is a component of an $\mathrm{SU}(2)_{R}$ triplet scalar which is responsible for the breaking of the left-right model into the SM. Note that we have stayed in the minimal picture in terms of the total field content; the model does not have any extra matter fields or any $\mathrm{SO}(10)$ multiplets in the scalar content other than the ones required to begin with.

The colored scalar in our set-up effectively induces the interaction terms of $\mathcal{S}$ with gluons and photons that lead to a diphoton final state after being produced via gluon fusion. In addition to the $\gamma \gamma$ decay, $\mathcal{S}$ can also decay to $j j, \gamma Z$, and $Z Z$ modes. We present the exclusion region in $M_{\chi}-\kappa$ plane for a benchmark resonance mass $M_{\mathcal{S}}=1 \mathrm{TeV}$ using the latest LHC data. We find that the most stringent bounds on the parameter space of our model come from the diphoton resonance search data. Therefore, we consider the diphoton channel as the most promising channel for the discovery of $\mathcal{S}$ at the LHC. As a prospect study, we compute the higher-luminosity LHC discovery reach of $\mathcal{S}$ by using a state-of-the-art multivariate technique. We present $5 \sigma$ discovery contours for different $\kappa$ choices in the $M_{\mathcal{S}}-M_{\chi}$ plane at the $13 \mathrm{TeV}$ LHC with 100 and $300 \mathrm{fb}^{-1}$ integrated luminosity. From our analysis, we find that for $\kappa \sim 1, M_{\mathcal{S}} \sim 0.5-2 \mathrm{TeV}$ and $M_{\chi} \sim 1 \mathrm{TeV}$ can easily be observed with $5 \sigma$ confidence level at the $13 \mathrm{TeV}$ LHC with $300 \mathrm{fb}^{-1}$ integrated luminosity. Note that the role of various systematic uncertainties is always important to consider in an analysis for robust and accurate prediction. But in the current scope, we do not consider systematic uncertainties for simplicity.

The unification of the couplings in the model is successfully realized, where the $\mathrm{TeV}$-scale colored triplet plays an important role. As discussed in [23], it is very difficult to achieve a successful $\mathrm{SO}(10)$ grand unification set-up with a TeV-scale left-right model. Slightly modifying the low energy scalar content by allowing a number of colored scalars, originated from various Pati-Salam multiplets, to become light generates the possibility of accommodating a TeV-scale left-right model in the $\mathrm{SO}(10)$ grand unification framework. Among a number of low energy scalar configurations, the ones with the very color-triplet selected in our model appear to particularly stand out [23]. We also note that the values obtained for the intermediate scale (where the Pati-Salam is broken) and the unification scale are sufficiently high to remain compatible with the experimental constraints regarding the leptoquark induced effects and the proton decay.

\section{Conflicts of Interest}

The authors declare that there are no conflicts of interest regarding the publication of this paper.

\section{Acknowledgments}

The authors would like to thank Ilia Gogoladze for stimulating conversations. This work is supported by the Swedish Research Council under Contract 621-2011-5107. Tanumoy 


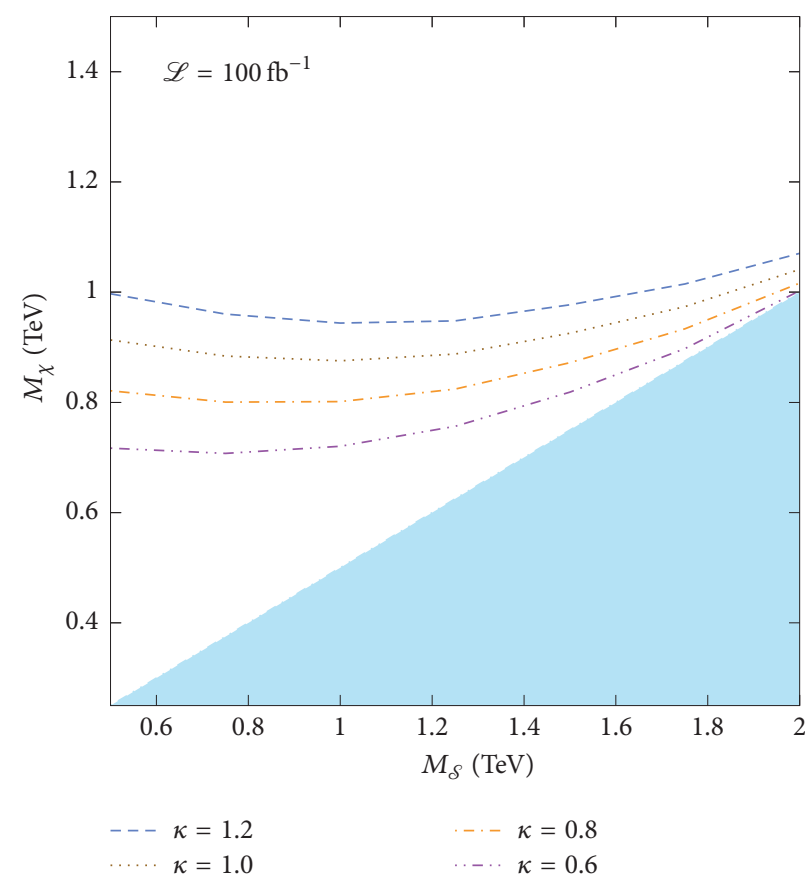

(a)

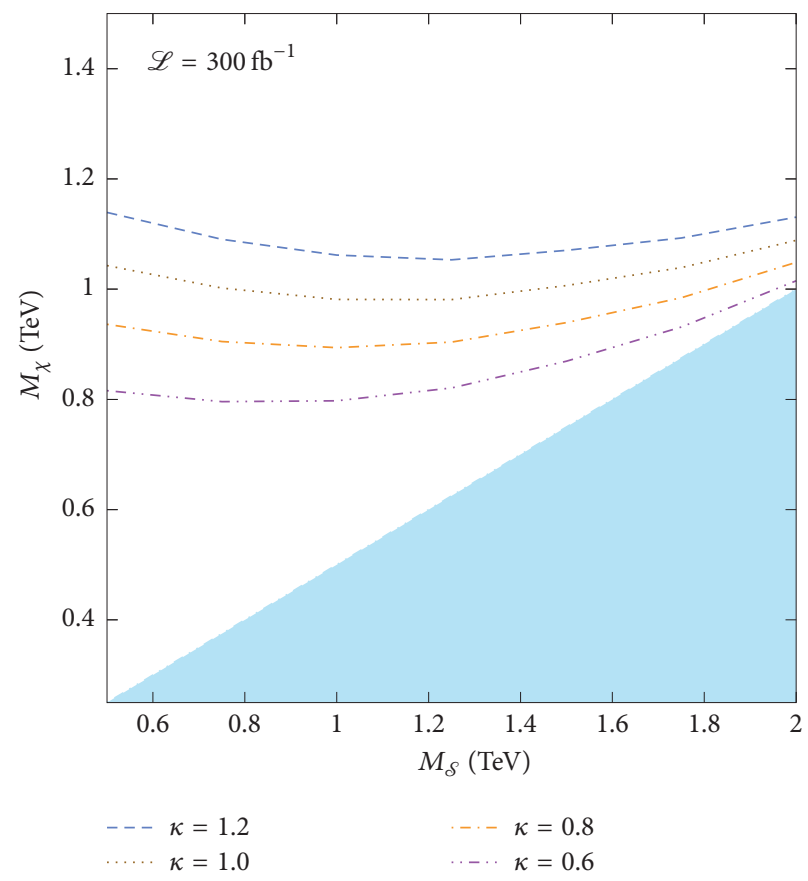

(b)

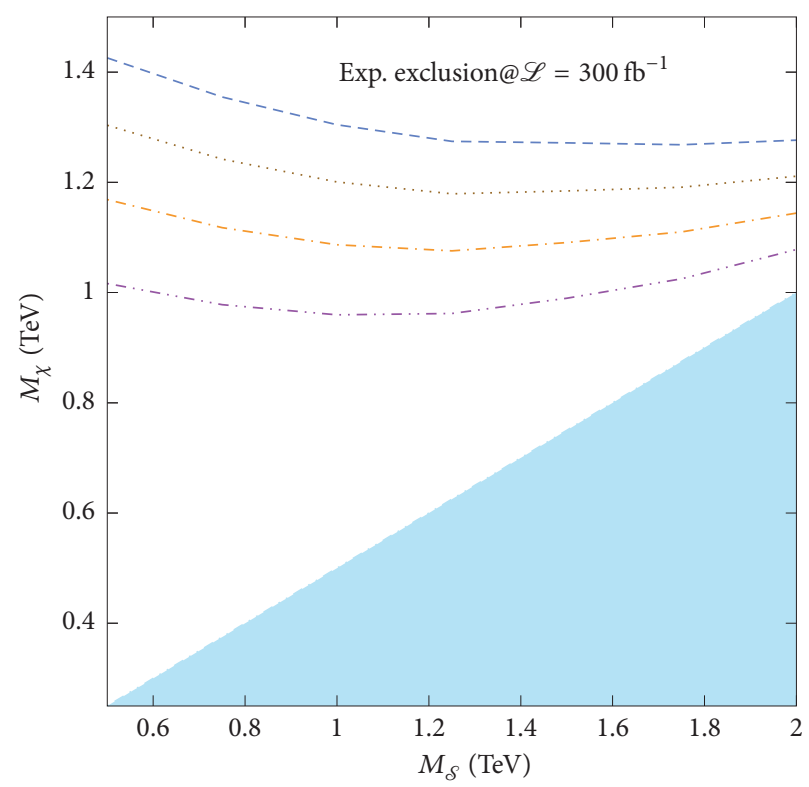

(c)

Figure 7: The $5 \sigma$ discovery contours in $M_{\delta}-M_{\chi}$ plane for different $\kappa$ at $13 \mathrm{TeV}$ LHC for (a) $100 \mathrm{fb}^{-1}$ and (b) $300 \mathrm{fb}^{-1}$ integrated luminosity. (c) The expected $2 \sigma$ exclusion plot for $\mathscr{L}=300 \mathrm{fb}^{-1}$. The sky-blue region cannot be probed in our analysis as we assume $M_{\chi}>M_{\mathcal{\delta}} / 2$.

Mandal is supported by the Carl Trygger Foundation under Contract CTS-14:206.

\section{References}

[1] S. Chatrchyan et al., "Observation of a new boson at a mass of $125 \mathrm{GeV}$ with the CMS experiment at the LHC," Physics Letters $B$, vol. 716, pp. 30-61, 2012.
[2] G. Aad et al., "Observation of a new particle in the search for the standard model higgs boson with the ATLAS detector at the LHC," Physics Letters B, vol. 716, pp. 1-29, 2012.

[3] M. Aaboud et al., "Search for resonances in diphoton events at $\sqrt{ } s=13 \mathrm{TeV}$ with the ATLAS detector," Journal of High Energy Physics, vol. 9, no. 1, 2016.

[4] V. Khachatryan et al., "Search for resonant production of highmass photon pairs in proton-proton collisions at $\sqrt{ } s=8$ and 
13 TeV," Physical Review Letters, vol. 117, no. 5, Article ID 051802, 2016.

[5] A. Strumia, "Interpreting the $750 \mathrm{GeV}$ digamma excess: a review," 2016, arXiv:1605.09401.

[6] G. Aad et al., "Search for high-mass diboson resonances with boson-tagged jets in proton-proton collisions at $\sqrt{ } s=8 \mathrm{TeV}$ with the ATLAS detector," Journal of High Energy Physics, vol. 12, no. 55, 2015.

[7] G. Aad et al., "Search for resonant diboson production in the $\ell \ell q \bar{q}$ final state in $p p$ collisions at $\sqrt{s}=8 \mathrm{TeV}$ with the ATLAS detector," The European Physical Journal C, vol. 75, no. 69, 2015.

[8] G. Aad et al., "Search for production of WW/WZ resonances decaying to a lepton, neutrino and jets in pp collisions at $\sqrt{ } s=$ $8 \mathrm{TeV}$ with the ATLAS detector," The European Physical Journal C, vol. 75, no. 5, p. 209, 2015.

[9] D. Chang, R. N. Mohapatra, and M. K. Parida, "Decoupling of parity- and $\mathrm{SU}(2)_{R}$-breaking scales: a new approach to left-right symmetric models," Physical Review Letters, vol. 52, no. 13, pp. 1072-1075, 1984.

[10] D. Chang, R. N. Mohapatra, and M. K. Parida, "New approach to left-right-symmetry breaking in unified gauge theories," Physical Review D, vol. 30, no. 5, article 1052, 1984.

[11] D. Chang, R. N. Mohapatra, J. M. Gipson, R. E. Marshak, and M. K. Parida, "Experimental tests of new SO(10) grand unification," Physical Review D, vol. 31, no. 7, pp. 1718-1732, 1985.

[12] M. K. Parida and C. C. Hazra, "Superheavy-Higgs-scalar effects in effective gauge theories from $\mathrm{SO}(10)$ grand unification with low-mass right-handed gauge bosons," Physical Review D, vol. 40, no. 9, pp. 3074-3085, 1989.

[13] N. G. Deshpande, E. Keith, and P. B. Pal, "Implications of the CERN LEP results for SO(10) grand unification," Physical Review D, vol. 46, no. 5, pp. 2261-2264, 1992.

[14] B. Bajc, A. Melfo, G. Senjanović, and F. Vissani, "Yukawa sector in nonsupersymmetric renormalizable $\mathrm{SO}(10)$," Physical Review D - Particles, Fields, Gravitation and Cosmology, vol. 73, no. 5, Article ID 055001, 2006.

[15] S. Bertolini, L. Di Luzio, and M. Malinský, "Intermediate mass scales in the nonsupersymmetric $\mathrm{SO}(10)$ grand unification: A reappraisal," Physical Review D - Particles, Fields, Gravitation and Cosmology, vol. 80, no. 1, Article ID 015013, 2009.

[16] K. S. Babu and R. N. Mohapatra, "Coupling unification, GUT-scale baryogenesis and neutron-antineutron oscillation in SO(10)," Physics Letters, 2012.

[17] R. L. Awasthi, M. K. Parida, and S. Patra, "Neutrino masses, dominant neutrinoless double beta decay, and observable lepton flavor violation in left-right models and $\mathrm{SO}(10)$ grand unification with low mass $W_{R}, Z_{R}$ bosons," Journal of High Energy Physics, vol. 2013, no. 8, article 122, 2013.

[18] B. P. Nayak and M. K. Parida, "New mechanism for Type-II seesaw dominance in $\mathrm{SO}(10)$ with low-mass $\mathrm{ZI}, \mathrm{RH}$ neutrinos, and verifiable LFV, LNV and proton decay," European Physical Journal C, vol. 75, article no. 183, 2015.

[19] M. K. Parida, R. L. Awasthi, and P. K. Sahu, "Proton decay and new contribution to $0 v 2 \beta$ decay in $\mathrm{SO}(10)$ with low-mass $Z^{\prime}$ boson, observable n-n oscillation, lepton flavor violation, and rare kaon decay," Journal of High Energy Physics, vol. 1, no. 45, 2015.

[20] T. D. Brennan, “Two loop unification of non-SUSY SO(10) GUT with TeV scalars," Physical Review D, vol. 95, no. 6, Article ID 065008, 2017.
[21] U. Aydemir, D. Minic, C. Sun, and T. Takeuchi, "Pati-salam unification from noncommutative geometry and the TeV-scale $W_{R}$ boson," International Journal of Modern Physics A, vol. 31, no. 1, Article ID 1550223, 2016.

[22] T. Bandyopadhyay, B. Brahmachari, and A. Raychaudhuri, "Implications of the CMS search for WR on grand unification," Journal of High Energy Physics, vol. 2, no. 23, 2016.

[23] U. Aydemir, "SO(10) grand unification in light of recent LHC searches and colored scalars at the TeV-scale," International Journal of Modern Physics A, vol. 31, no. 8, Article ID 1650034, 2016.

[24] M. K. Parida, B. P. Nayak, R. Satpathy, and R. L. Awasthi, "Standard coupling unification in $\mathrm{SO}(10)$, hybrid seesaw neutrino mass and leptogenesis, dark matter, and proton lifetime predictions," Journal of High Energy Physics, vol. 2017, no. 75, 58 pages, 2017, arXiv:1608.03956.

[25] N. G. Deshpande, E. Keith, and T. G. Rizzo, "SO(10) grand unification with a low-energy $\mathrm{SU}(2)_{R}$-symmetry-breaking scale $M_{R}$," Physical Review Letters, vol. 70, no. 21, pp. 3189-3192, 1993.

[26] T. Fukuyama, A. Ilakovac, T. Kikuchi, S. Meljanac, and N. Okada, "General formulation for proton decay rate in minimal supersymmetric SO(10) GUT," The European Physical Journal C, vol. 42, pp. 191-203, 2005.

[27] T. Fukuyama, A. Ilakovac, T. Kikuchi, S. Meljanac, and N. Okada, "SO(10) group theory for the unified model building," Journal of Mathematical Physics, vol. 46, no. 3, Article ID 033505, 2005.

[28] S. K. Majee, M. K. Parida, A. Raychaudhuri, and U. Sarkar, "Low intermediate scales for leptogenesis in supersymmetric $\mathrm{SO}(10)$ grand unified theories," Physical Review D, vol. 75, no. 7, Article ID 075003, 2007.

[29] M. K. Parida, "Intermediate left-right gauge symmetry, unification of couplings and fermion masses in SUSY SO(10)XS(4)," Physical Review D, vol. 78, no. 5, Article ID 053004, 2008.

[30] P. S. B. Dev and R. N. Mohapatra, "TeV scale inverse seesaw model in $\mathrm{SO}(10)$ and leptonic nonunitarity effects," Physical Review D, vol. 81, Article ID 013001, 2010.

[31] M. K. Parida and A. Raychaudhuri, "Inverse seesaw mechanism, leptogenesis, observable proton decay, and $\Delta_{R}^{ \pm \pm}$in supersymmetric SO(10) with heavy $W_{R}$," Physical Review $D$, vol. 82, no. 9, Article ID 093017, 2010.

[32] J. C. Pati and A. Salam, "Erratum: Lepton number as the fourth 'color," Physical Review D, vol. 10, pp. 275-289, 1975.

[33] R. N. Mohapatra and J. C. Pati, "Natural' left-right symmetry," Physical Review D, vol. 11, no. 9, pp. 2558-2561, 1975.

[34] G. Senjanovic and R. N. Mohapatra, "Exact left-right symmetry and spontaneous violation of parity," Physical Review D, vol. 12, no. 5, pp. 1502-1505, 1975.

[35] R. N. Mohapatra and G. Senjanovic, "Neutrino mass and spontaneous parity nonconservation," Physical Review Letters, vol. 44, p. 912, 1980.

[36] R. N. Mohapatra and G. Senjanović, "Neutrino masses and mixings in gauge models with spontaneous parity violation," Physical Review D, vol. 23, article 165, 1981.

[37] P. Duka, J. Gluza, and M. Zralek, "Quantization and renormalization of the manifest left-right symmetric model of electroweak interactions," Annals of Physics, vol. 280, no. 2, pp. 336408, 2000.

[38] U. Aydemir, D. Minic, and T. Takeuchi, "The Higgs mass and the emergence of new physics," Physics Letters, Section B: Nuclear, Elementary Particle and High-Energy Physics, vol. 724, no. 4-5, pp. 301-305, 2013. 
[39] U. Aydemir, D. Minic, C. Sun, and T. Takeuchi, "Higgs mass, superconnections, and the $\mathrm{TeV}$-scale left-right symmetric model," Physical Review D - Particles, Fields, Gravitation and Cosmology, vol. 91, no. 4, Article ID 045020, 2015.

[40] F. Del Aguila and L. E. Ibáñez, "Higgs bosons in $\mathrm{SO}(10)$ and partial unification," Nuclear Physics, Section B, vol. 177, no. 1, pp. 60-86, 1981.

[41] A. Maiezza, M. Nemevšek, F. Nesti, and G. Senjanović, "Leftright symmetry at LHC," Physical Review D - Particles, Fields, Gravitation and Cosmology, vol. 82, no. 5, Article ID 055022, 2010.

[42] P. Minkowski, “ $\mu \rightarrow \mathrm{e} \gamma$ at a rate of one out of $10^{9}$ muon decays?” Physics Letters B, vol. 67, pp. 421-428, 1977.

[43] M. Gell-Mann, P. Ramond, and S. Richard, "Complex spinors and unified theories," Conf. Proc, Article ID C790927, pp. 315321, 1979, https://arxiv.org/abs/1306.4669.

[44] T. Yanagida, "Horizontal symmetry and masses of neutrinos," Conf. Proc, Article ID C7902131, pp. 95-99, 1979.

[45] J. Schechter and J. W. F. Valle, "Neutrino masses in $\mathrm{SU}(2) \otimes \mathrm{U}(1)$ theories," Physical Review D, vol. 22, p. 2227, 1980.

[46] J. Schechter and J. W. F. Valle, "Neutrino decay and spontaneous violation of lepton number," Physical Review D, vol. 25, p. 774, 1982.

[47] C. Patrignani et al., "Review of particle physics," Chinese Physics C, vol. 40, no. 10, Article ID 100001, 2016.

[48] S. Schael et al., "Precision electroweak measurements on the $\mathrm{Z}$ resonance," Physics Reports, vol. 427, no. 5-6, pp. 254-457, 2006.

[49] D. R. T. Jones, "The two loop beta function for a G(1) x G(2) gauge theory," Physical Review D, vol. 25, no. 581, 1982.

[50] M. Lindner and M. Weiser, "Gauge coupling unification in leftright symmetric models," Physics Letters. B. Particle Physics, Nuclear Physics and Cosmology, vol. 383, no. 4, pp. 405-414, 1996.

[51] J. L. Evans, N. Nagata, K. A. Olive, and J. Zheng, "The ATLAS diboson resonance in non-supersymmetric $\mathrm{SO}(10)$," Journal of High Energy Physics, vol. 2016, no. 2, article no. 120, pp. 1-25, 2016.

[52] S. Bertolini, L. Di Luzio, and M. Malinský, "Seesaw scale in the minimal renormalizable SO(10) grand unification," Physical Review D - Particles, Fields, Gravitation and Cosmology, vol. 85, no. 9, Article ID 095014, 2012.

[53] G. R. Dvali, "Light colour-triplet Higgs is compatible with proton stability: an alternative approach to the doublet-triplet splitting problem," Physics Letters B, vol. 372, no. 1-2, pp. 113-120, 1996.

[54] S. Rakshit, G. Raz, S. Roy, and Y. Shadmi, "Light grand unified theory triplets and Yukawa splitting," Physical Review D, vol. 69, no. 9, Article ID 095006, 2004.

[55] V. Tello, M. Nemevek, F. Nesti, G. Senjanović, and F. Vissani, "Left-right symmetry: from the LHC to neutrinoless double beta decay," Physical Review Letters, vol. 106, no. 15, Article ID 151801, 2011.

[56] R. N. Mohapatra, "Neutrino mass as a signal of TeV scale physics," Nuclear Physics B, vol. 908, pp. 423-435, 2016.

[57] J. C. Helo, M. Hirsch, and S. Kovalenko, "Erratum: Heavy neutrino searches at the LHC with displaced vertices [Phys. Rev. D 89, 073005 (2014)]," Physical Review D, vol. 93, no. 9, Article ID 099902, 2016.

[58] R. Ruiz, Lepton number violation at colliders from kinematically inaccessible gauge bosons, arXiv:1703.04669 hep-ph.
[59] A. Alloul, N. D. Christensen, C. Degrande, C. Duhr, and B. Fuks, "FeynRules 2.0-a complete toolbox for tree-level phenomenology," Computer Physics Communications, vol. 185, no. 8, pp. 2250-2300, 2014.

[60] J. Alwall, R. Frederix, and S. Frixione, "The automated computation of tree-level and next-to-leading order differential cross sections, and their matching to parton shower simulations," Journal of High Energy Physics, vol. 2014, no. 7, article 079, 2014.

[61] J. Pumplin, D. R. Stump, J. Huston, H.-L. Lai, P. Nadolsky, and W.-K. Tung, "New generation of Parton distributions with uncertainties from global QCD analysis," Journal of High Energy Physics, vol. 7, no. 12, 2002.

[62] The ATLAS collaboration, "Search for scalar diphoton resonances with $15.4 \mathrm{fb}^{-1}$ of data collected at $\sqrt{s}=13 \mathrm{TeV}$ in 2015 and 2016 with the ATLAS detector," ATLAS-CONF-2016-059, 2016.

[63] V. Khachatryan et al., "Search for high-mass diphoton resonances in proton-proton collisions at $13 \mathrm{TeV}$ and combination with $8 \mathrm{TeV}$ search," Physics Letters B, vol. 767, pp. 147-170, 2017.

[64] CMS Collaboration, "Search for high-mass resonances in $Z \gamma \rightarrow$ $e^{+} e^{-} \gamma / \mu^{+} \mu^{-} \gamma$ final states in proton-proton collisions at $\sqrt{s}=$ 13 TeV," CMS-PAS-EXO-16-034.

[65] CMS Collaboration, "Search for high-mass resonances in $\mathrm{Z}$ (qq) $\gamma$ final state in pp collisions at $\sqrt{s}=13 \mathrm{TeV}$ with $12.9 \mathrm{fb}^{-1}$," CMS-PAS-EXO-16-035.

[66] ATLAS collaboration, "Searches for heavy ZZ and ZW resonances in the llqq and $\nu \nu$ qq final states in pp collisions at $\sqrt{s}=13 \mathrm{TeV}$ with the ATLAS detector," ATLAS-CONF-2016082, 2016.

[67] The ATLAS collaboration, "Search for New Phenomena in Dijet Events with the ATLAS Detector at $\sqrt{s}=13 \mathrm{TeV}$ with 2015 and 2016 data," ATLAS-CONF-2016-069, 2016.

[68] CMS Collaboration, "Searches for narrow resonances decaying to dijets in proton-proton collisions at $13 \mathrm{TeV}$ using 12.9 inverse femtobarns," CMS-PAS-EXO-16-032.

[69] D. de Florian et al., "Handbook of lhc higgs cross sections: 4. deciphering the nature of the higgs sector," Tech. Rep., 2016, arXiv:1610.07922 hep-ph.

[70] T. Sjöstrand, S. Mrenna, and P. Skands, "PYTHIA 6.4 physics and manual," Journal of High Energy Physics, vol. 5, article 026, 2006.

[71] J. de Favereau, C. Delaere, P. Demin et al., "DELPHES 3: a modular framework for fast simulation of a generic collider experiment," Journal of High Energy Physics, vol. 2014, article 57, 2014.

[72] M. Cacciari, G. P. Salam, and G. Soyez, "FastJet user manual: (For version 3.0.2)," European Physical Journal C, vol. 72, no. 3, article 1896, pp. 1-54, 2012.

[73] M. Cacciari, G. P. Salam, and G. Soyez, "The Anti-k(t) jet clustering algorithm," Journal of High Energy Physics, vol. 2008, article 063, 2008.

[74] A. Hocker et al., "TMVA - toolkit for multivariate data analysis," in Proceedings of PoS ACAT, vol. 40, 2007.

[75] M. L. Mangano, M. Moretti, F. Piccinini, and M. Treccani, "Matching matrix elements and shower evolution for top-pair production in hadronic collisions," Journal of High Energy Physics, vol. 2007, no. 1, article no. 013, 2007. 

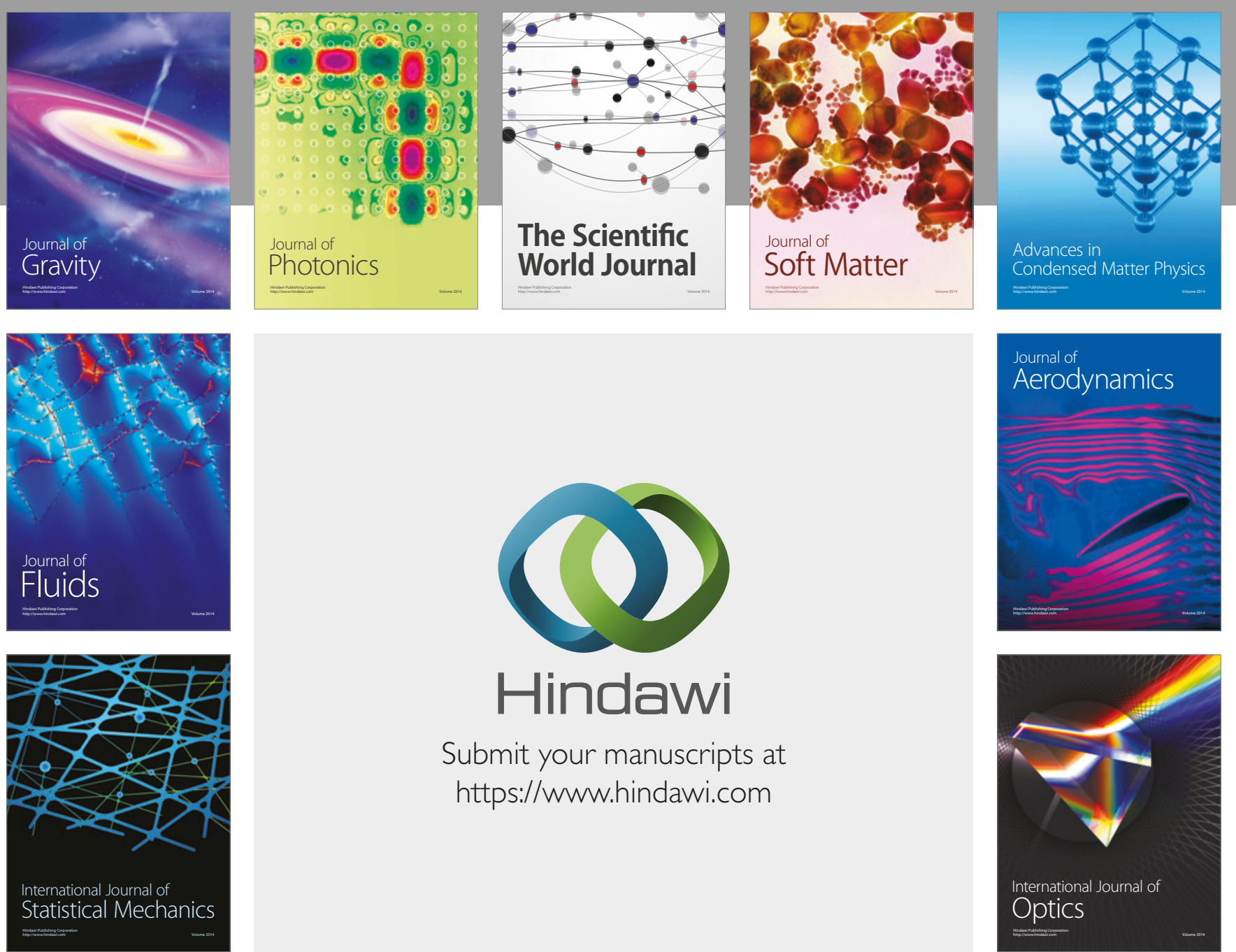

Submit your manuscripts at

https://www.hindawi.com
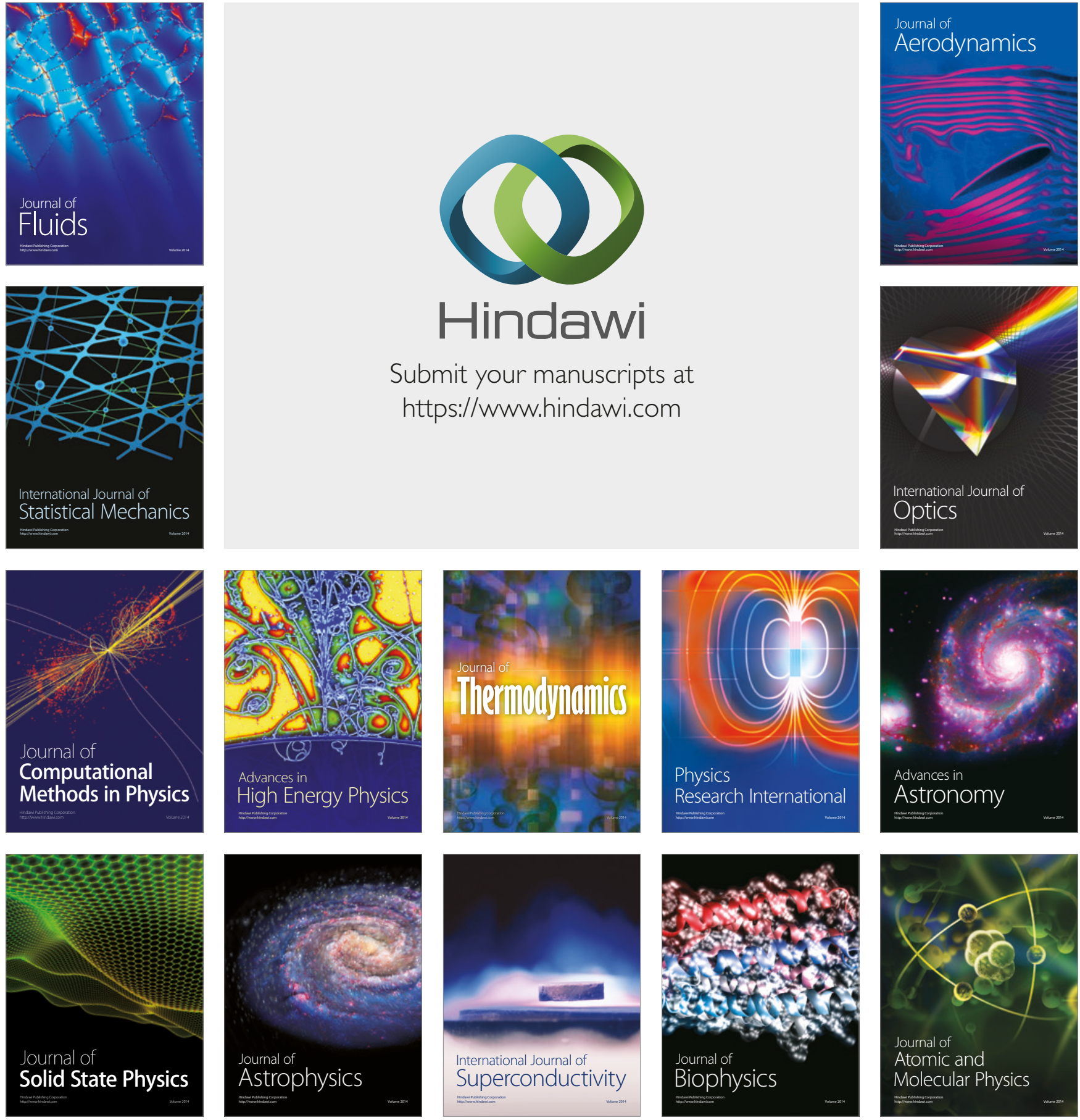